\title{
Some open questions in the management of mineralization by organisms \\ PETER FRATZL
}

Max Planck Institute of Colloids and Interfaces

Presenting Author: fratzl@mpikg.mpg.de

Biomineralization is a central process for many organisms to synthesize protective shells or skeletal elements. It is also important in the context of the homeostasis of essential ions, for which mineral can be a repository. Over the last decades, there was significant progress in the understanding of crystal nucleation and growth in many organisms, including the role of (amorphous) precursors as well as ionic and macromolecular additives. Mineral deposition involves a change of local ion concentrations from millimolar in typical body fluids to molar in the mineralized structures. This implies that preventing nucleation and precipitation in unwanted areas, as well as the transport of ions and precursors to the site of mineral formation are aspects as crucial for biomineralization as directed nucleation and growth. Rather little is known about the transport processes that might also be very different in diverse organisms. Recent observations for some aquatic animals that ions may be extracted directly from seawater cannot possibly apply to terrestrial vertebrates. Based on recent analyses of osteocyte networks in bone, informed hypotheses will be formulated in this talk about their potential role in the transport of mineral precursors. 